\title{
PENILE STRANGULATION BY A HARD PLASTIC BOTTLE NECK: A CASE REPORT
}

N. Lingaraju, Arunkumar. U, Zaheeruddin, Sajjan Dundappa, Sali. S. S.
1. Assistant professor, Dept. of General surgery, ESIC Medical College. Gulbarga. Karnataka.
2. Assistant professor, Dept. of General surgery, ESIC Medical College. Gulbarga. Karnataka.
3. Senior resident, Dept. of General surgery, ESIC Medical College. Gulbarga. Karnataka.
4. Senior Specialist, Dept. of General surgery, District hospital. Gulbarga.
5. Senior Specialist, Dept. of General surgery, District hospital. Gulbarga.

\author{
CORRESPONDING AUTHOR: \\ Dr. Lingaraju. N, \\ C/O Shivaleela, plot no.12, \\ Near hanuman temple, \\ Jayanagar, sedam road, \\ Gulbarga. Karnataka. \\ E-mail: lingu1983@yahoo.com
}

\begin{abstract}
Application of Various metallic and non-metallic constricting objects on the external male genitalia for increasing sexual performance or because of autoerotic intentions is an unusual practice that can potentially lead to penile strangulation with severe consequences. To remove such an object is a challenge as the chances of injury to the surgeon and the patient were high. Here with we report a 65 year old male who presented in our hospital with a hard plastic bottle neck at the base of penis which led to penile strangulation. The constricting agent was successfully removed. The patient had an uneventful recovery.
\end{abstract}

KEY WORDS: Penile strangulation, Penile gangrene, Plastic bottle neck, Metallic ring.

INTRODUCTION: Penile strangulation by various object s (e.g., wedding rings, rubber bands, plumbing metal ring, plastic bottle neck, steel bearing, etc.)[1-3] presents a situation that challenges the surgeon to be innovative in selecting the treatment. In adults these constricting bands, whether expandable or non-expandable, are placed deliberately by the person himself for masturbation or by the female counterpart to prolong erection [4] .In children these are used to prevent enuresis and incontinence or as an innocent childish experiment. We report a case, which had hard plastic bottleneck as a constricting agent, which was successfully removed.

CASE REPORT: A 65 year old male presented to surgical OPD with markedly swollen penis. He had placed his penis in a hard plastic bottle for masturbation \& prolongation of sexual pleasure 24 hours ago. The bottleneck got stuck and constricted the base of the penis. He complained of pain and swelling on his external genitalia but he did not report any difficulty in passing urine. On examination, gross oedema of the penis, with bottleneck around the base of penis. There was no change in skin coloration or texture and no alteration of sensation distal constricting object (fig-1) 


\section{CASE REPORT}

After an initial attempt at removal with a surgical blade was unsuccessful due to the thick plastic bottle an orthopedic oscillating saw was utilized and removed successfully (fig-2).

There was no damage to the penis at the strangulation site as well as on the distal part. Penis was cleaned with povidone-iodine solution, an antibiotic ointment was applied and dressed with Vaseline gauze and bandage was applied and bladder catheterization done using $16 \mathrm{fr}$ Foleys catheter after the procedure (fig-3).

The patient remained cooperative during the procedure. Before starting the procedure the patient was pre medicated with atropine and diclofenac $75 \mathrm{mg}$. Antibiotics and tetanus prophylaxis was also given.

DISCUSSION: The first case of a foreign body externally applied to the penis was reported in 1755 by Gauthier [5] .Penile strangulation injuries range from simple penile engorgement to ulceration, necrosis, urinary fistula or even gangrene.

A variety of metallic and non-metallic rings causing constriction the external genitalia has been described in literature [6]. The motivation for intentional placement of penile constriction devices and the type of foreign body is variable depending on the patient's age. The adult population frequently reports erotic or autoerotic goals when intentionally placing constricting devices [7]. Pediatric patients may present with either accidental or intentional placement of a strangulating object, most commonly strands of hair [8]. The most often reported cause of children, or their guardians, intentionally placing hair around the penis is to prevent enuresis.

AL Bhat et al (1999) graded these injuries as follows [9]

GRADE I: $\quad$ Edema of distal penis. No evidence of skin ulceration or urethral injury

GRADE II Injury to skin and constriction of corpus spongiosum but no evidence of urethral injury. Distal penile edema with decreased penile sensation.

GRADE III: Injury to skin and urethra but no urethral fistula. Loss of distal penile sensations.

GRADE IV: Complete division of Corpus spongiosum leading to urethral fistula and constriction of corpus cavernosa with loss of distal penile sensations.

GRADE V: $\quad$ Gangrene, necrosis, or complete amputation of distal penis

The effects of penile entrapment depend on the interval of foreign body attached to the penis. Although gangrene has been reported, it is uncommon. Because each corpus cavernosum has an individual artery, and the thickness of Buck's fascia and corporeal tissue resists pressure on the deep vessels [10] The skin devoid of subcutaneous tissue located most superficially, is affected first. Complications of penile strangulation can be local and systemic.

Local complications include minor ones like venous engorgement due to impaired venous return, and the necrosis of penile skin from prolonged Pressure. More significant local complications include penile gangrene from prolonged vascular ischemia which may require amputation as a lifesaving measure, and the formation of urethra-cutaneous fistula [11]. Systemic complications are less well documented in the literature. Renal impairment (obstructive uropathy) from the obstruction is one such complication [12] 
Numerous methods have been described for removal of constricting devices. When choosing a method one must take into account the material to be removed, severity of penile injury, and availability of tools. With metallic bands that cannot be removed with conventional ring cutters the string technique in concert with penile aspiration may be utilized [13]. Power driven cutting tools include dremmel saws [14] and oscillating orthopedic saws [15] have been employed with excellent results, often avoiding the need for surgical intervention. However, if the penis is gangrenous, necrotic, or other modalities have failed, degloving, or amputation of the penis may be indicated contingent on the extent of devitalized tissue.

CONCLUSION: Penile strangulation is a urologic emergency with potentially severe clinical consequences. Aim should be restoration of blood supply and micturition with least complications There is no standard protocol mentioned to deal with such cases. Every case needs individual approach depending on the circumstances and facilities available. This case highlights the successful use of an orthopedic oscillating saw to remove a constriction ring.

ACKNOWLEDGEMENT: I/We would like to express our special thanks to Dr.M.R.Chandrashekar, Dean \& Director ESIC Medical college Gulbarga, Dr.Nalini namoshi ,District surgeon, Dr.Asif ali, RMO, Dr.Dhadve, OPD surgeon, District hospital Gulbarga, Dr.Biradar.V.B, Dr.Harsoor.M.S, Professors of surgery ESIC Medical college Gulbarga \& Dr.B.N.Joshi, Dr.Ranganath Katti, Dr.M.R.Joshi, senior specialists District hospital Gulbarga For allowing us to utilize the services of District hospital Gulbarga and guiding us in writing this paper

\section{REFERENCES:}

1. McLaughlin T, Coyner W, Removal of a strangulating metal bearing from the penis. J Urol 1989. 141(3): p.617

2. Perabo FG, Steiner G, Albers P, Müller SC ,Treatment of penile strangulation caused by constrictions devices. Urology 2002. 59 (1): p.137

3. Kadio glu A, Cayan S, Ozcan F, Tellalo g lu S (Treatment of penile incarceration in an impotent patient. Int Urol Nephrol 1995. 27 (5): p.639 -641.

4. Bhat AL, Kumar A, Mathur SC, Gangwal K. Penile strangulation. Br J Urology 1991; 68: p.61821.

5. 5 Gauthier M. Observation d'un e'tranglement des testicules et de la verge, occasionn'e par le passage d'un briquet, J Med Chir Pharmacol 1755. 3: p.358.

6. Perabo F, Gabriel S, Albers P, Muller S: Treatment of Penile strangulation caused by constricting devices. Urology 2002, 59(1): p.137-139.

7. J. Silberstein, J. Gr abowski, C. Lakin, and I . Goldstein, "Penile constriction devices: case report, review of the literature, and re commendations for extrication," Journal of Sexual Medicine, 2008. 5(7) ; p.1747-1757,

8. B. Sing h, H. Ki m, and S. H. Wax, "Strangulation of glans penis by hair," Ur o l o g y, 1978 11(2); p.170-172,.

9. Bhat AL, Kumar A, Mathur SC, Gangwal K. Penile strangulation. Br J Urology 1991.68; p 61821. 


\section{CASE REPORT}

10. Mohr AM, Pham AM, Lavery RF, et al. Management of trauma to male external genitalia: the usefulness of American association for the surgery of trauma organ injury scales. J Urology 2003; 170: p.2311-5.

11. Kore RN, Blacklock AR,. Ring the fire brigade. Br. J. Urol.1996. 78; p.948.

12. Huang JK, Holt D, Philip T (). Penile constriction by foreign bodies: the use of a dental drill. Br. J. Urol. 1997.79; p.801.

13. J.Noh, T.W.Kang, T.Heo, D.D. Kwon, et al "Penile strangulation treated with the modified string method, " Ur o lo g y ,2004. 64(3); p.591.

14. S. Bro ck and W. Kuhn, "Removal of constricting bands using the Dremel drill," Academic Emergency Medicine, 1999 .6(11); p.1182-1183,.

15. M .May, S. Gunia, C. Helke, et al, "Penile entrapment in a plastic bottle-a case for using an oscillating splint saw," International Urolog

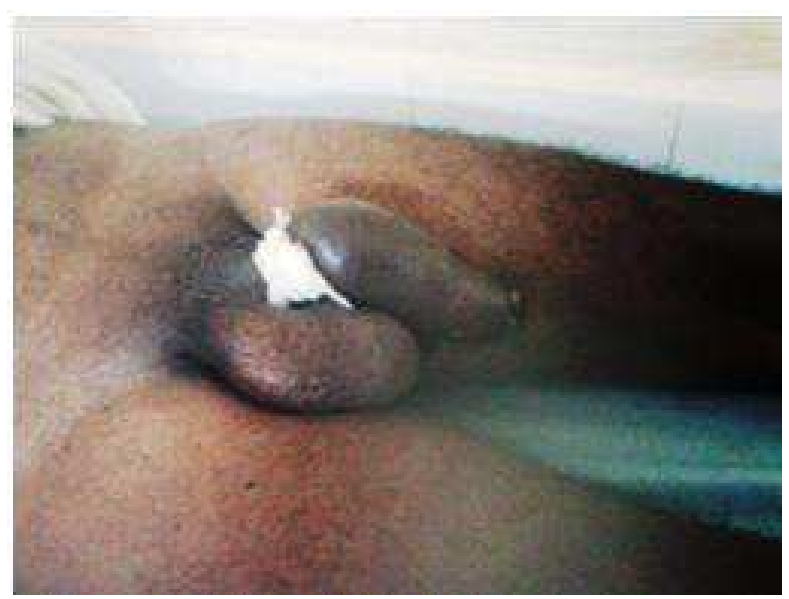

FIG-1: Oedematous penis with the constricting agent in-situ.

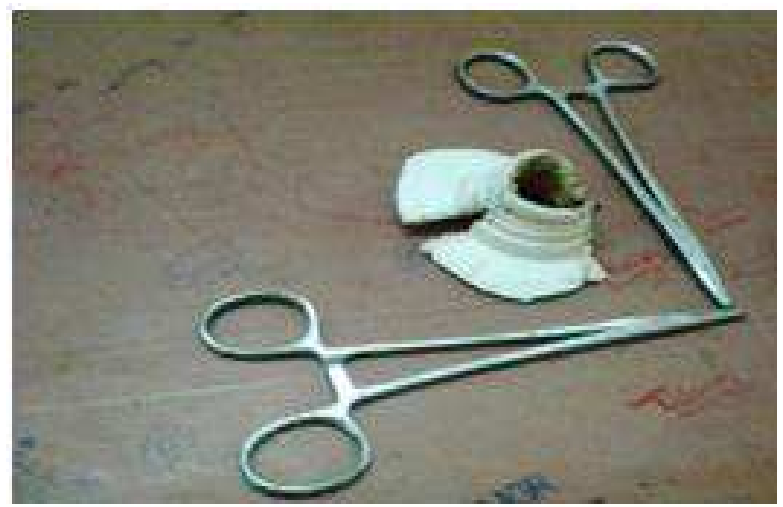

FIG-2: Constricting agent which was removed 


\section{CASE REPORT}

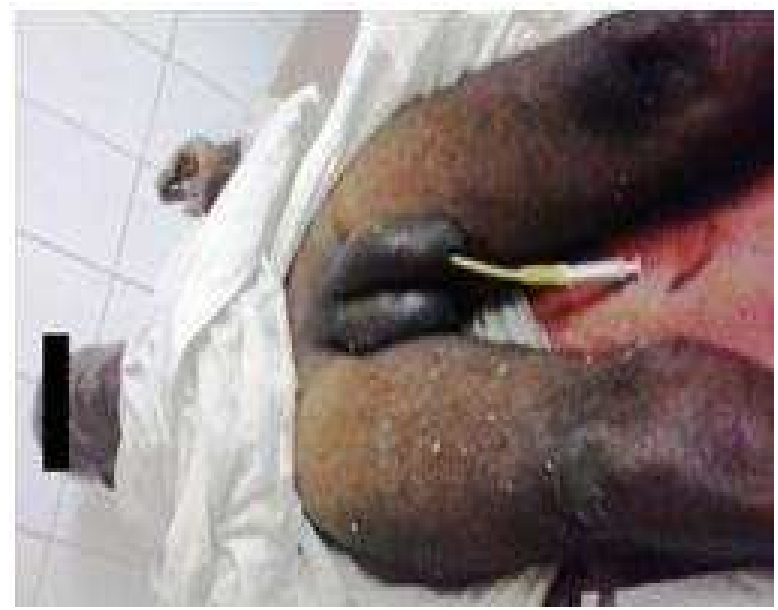

FIG-3: After the removal of constricting agent \& Cathetrising the bladder. 